[CONTRIBUTION FROM THE LABORATORY OH TUIE LORMAMNA SLGAR FARERIMENT STATION, NEW ORLEAN- I.A.]

\title{
ON THE DETERMINATION OF REDUCING SUGARS.
}

\author{
BY Y. ZERBAN ANI W. P. NAQUiN. \\ Received May 1 , rgo:
}

Munson and Walker, ${ }^{1}$ in their method for the determination of reducing sugars, recommend the use of a porcelain Gooch and the weighing of the precipitate as cuprous oxide after drying for one-half hour. A determination made in this way requires from 35-40 minutes, and cannot, in regard to speed, compete with the volumetric method. It would therefore be desirable to shorten the time consumed by the manipulations without changing the method itself. We have carried out some experiments from this point of view, at the same time investigating some questions in regard to the determination of reducing sugars in low grade, sugar house products.

Pellet $^{2}$ was the first to suggest the use of Munroe-Neubauer ${ }^{3}$ crucibles instead of the ordinary Gooch for the determination of reducing sugars. This method would save the trouble of purifying asbestos and of preparing the filter, not a small item when a number of analyses are required. The Neubaner crucible offers the further advantage, that it can be heated to a red heat, while porcelain Gooch crucibles, when treated in this manner, often lose weight by the breaking off of small particles from the perforated bottom.

Several series of analyses were made using the method of Munson and Walker, but substituting Munroe-Neubauer crucibles for the ordinary Gooch. The crucibles used were secured from the Vereinigte Fabriken für Laboratoriumsbedarf, Berlin, and weighed 22 grams each. It was found that they serve the purpose very well, completely retaining the cuprous oxide, and allowing of very rapid filtration.

The experiments were made with an invert sugar solution prepared according to the directions given in Spencer's handbook for cane sugar manufacturers. The cuprous oxide precipitate was first weighed as such, after drying for 30 minutes in a water oven, and it was then converted into cupric oxide by heating in air or oxygen. In order to prevent the access of reducing flame gases to the material, the crucible was placed in a small platinum dish. The manufacturers also furnish crucibles with a platinum cap. In the first series of experiments the cuprous oxide was heated for to minutes over a Bunsen burner and the following results were obtained:

'THIS IOURNAL, 28,663.

"Bull assoc chim sucr. dist., 24, 1392.

" "A Platinum Crucible with a Filter of Spongy Platinum," Chem. Yeus, 58, 10 I (I888); IHLS JOtRNiL, 29,6,3.3. 


\begin{tabular}{cccc}
$\mathrm{Cu}_{2} \mathrm{O}$. & $\mathrm{CuO}$. & $\mathrm{Cu}$ from $\mathrm{Cu}_{2} \mathrm{O}$. & $\mathrm{Cu}$ from CuO \\
0.2700 & 0.2955 & 0.2396 & $0.236 \mathrm{I}$ \\
0.2700 & 0.2985 & 0.2396 & 0.2385 \\
0.2730 & 0.2970 & 0.2424 & 0.2363 \\
0.2760 & 0.3020 & 0.2450 & 0.2413 \\
0.2720 & 0.2990 & 0.2414 & 0.2389 \\
0.2710 & 0.2990 & 0.2406 & 0.2389 \\
0.2730 & 0.3010 & 0.2424 & 0.2404 \\
0.2695 & 0.2990 & 0.2392 & 0.2389 \\
0.2730 & 0.3010 & 0.2424 & 0.2404 \\
& \multicolumn{3}{c}{ Average, 0.2410} \\
& \multicolumn{3}{c}{ Difference, 0.0023}
\end{tabular}

As is seen from the table, the weight of copper calculated from the cupric oxide is in every case lower than that calculated from the cuprous oxide. That this is not due to incomplete oxidation of the cuprous oxide was proven by a second series of experiments where the cuprous oxide was heated for 30 minutes in a current of oxygen, with the following results:

$\begin{array}{cccc}\mathrm{Cu}_{2} \mathrm{O} . & \text { CuO. } & \mathrm{Cu} \text { from } \mathrm{Cu}_{2} \mathrm{O} . & \text { Cu from CuO } \\ 0.2648 & 0.2924 & 0.235 \mathrm{I} & 0.2336 \\ 0.2632 & 0.2902 & 0.2337 & 0.23 \mathrm{I} 8 \\ 0.2666 & 0.2936 & 0.2367 & 0.2345 \\ 0.2666 & 0.2936 & 0.2367 & 0.2345 \\ 0.2664 & 0.2934 & 0.2365 & 0.2344 \\ 0.2675 & 0.2957 & 0.2375 & 0.2362 \\ 0.2688 & 0.2948 & 0.2386 & 0.2354 \\ 0.2650 & 0.2920 & 0.2353 & 0.2333 \\ 0.2638 & 0.2920 & 0.2342 & 0.2333 \\ 0.2658 & 0.2950 & 0.2360 & 0.2357 \\ & & - & 0.2342\end{array}$

It is apparent that the heating in air for Io minutes over a bunsen burner is sufficient for a complete oxidation. The discrepancy of the results must therefore be due to some other cause. In order to arrive at a conclusion about this point, we had to determine the metallic copper in the precipitate. Several methods could be used for this purpose: reduction by hydrogen, electrolysis, or some volumetric method. The first method could not be used on account of the danger of the reduced copper forming an alloy with the platinum. Since we are not equipped for electrolytic determinations, Low's volumetric method was adopted for the purpose, with very satisfactory results.

Two series or to and 3 experiments, respectively, gave the following figures:

$\begin{array}{ccc}\mathrm{Cu} \text { from } \mathrm{Cu}_{2} \mathrm{O} . & \mathrm{Cu} \text { from CuO. } & \mathrm{Cu} \text { by Low. } \\ 0.2378 & 0.2359 & 0.2354 \\ 0.2285 & 0.2266 & 0.2265\end{array}$


The difference between the figures for copper as calculated from the cuprous oxide and from the copper oxide is o.oorg gram. The copper as determined by Low's method is nearly identical with the quantity calculated from the cupric oxide, but $0.0020-0.0024$ gram lower than that calculated from the cuprous oxide. This shows that in the method of procedure used, the weight of the cuprous oxide is a little too high. This is necessarily due to small amounts of a volatile substance which is not driven off at the temperature of the water oven. The excess weight is, however, so small that we did not attempt to determine the nature of this substance. In all probability it is nothing but water. This discrepancy is noticed only when Munroe-Neubauer crucibles are used, but not with the ordinary Gcoch, as Munson and Waiker have shown.

By, using Munroe-Neubauer crucibles and weighing the copper as black oxide, the method of Munson and Walker could be carried out in less than one-half of the time required for the original method. But the difference between the copper calculated from the cuprous and the cupric oxide prevents the direct use of Munson and Walker's tables. It was necessary to find out whether this difference changes with the quantity of the precipitate or whether it is a constant. Some experiments were made with varying amounts of invert sugar with the following results:

Cu from $\mathrm{Cu}_{2} \mathrm{O}$.
0.0799
0.0792
0.2285
0.2378
0.3641
0.4222
0.4229

$$
\begin{gathered}
\text { Cu from C:O. } \\
0.0783 \\
0.0772 \\
0.2266 \\
0.2359 \\
0.3626 \\
0.4205 \\
0.4212
\end{gathered}
$$

Diff.

$$
\begin{aligned}
& 0.0016 \\
& 0.0020 \\
& 0.0019 \\
& 0.0019 \\
& 0.0015 \\
& 0.0017 \\
& 0.0017
\end{aligned}
$$

$$
\text { Average Difference, } \quad 0.0017
$$

The difference is found to be constant and the correction to be applied is 0.0017 gram added to the weight of the copper calculated from the cupric oxide.

Our experiments were next extended to the analysis of a low-grade Louisiana molasses.

In the report on sugar presented at the Jamestown meeting of the Association of Official Agricultural Chemists, it was recommended by the referee to discontinue the use of lead subacetate solution previous to the determination of reducing sugars. It was definitely shown that this reagent, employed in any form, precipitates considerable quantities of reducing sugars and especially of levulose. The lower figures obtained for reducing sugars after clarification with lead subacetate are, there- 
fore, partly due to the removal of reducing sugars. But if the solution be not clarified at all, there is a danger of contaminating the precipitate with appreciable quantities of organic and mineral matter. For this reason the use of agents which do not act like lead subacetate was recommended, and normal lead acetate was named as one that may be used for this purpose.

This question of clarification was also studied in connection with the other experiments. In one series the solution was clarified by carefully adding neutral lead acetate solution until no more precipitate was produced; in the second series we did not clarify at all, but simply filtered the solution. In every case the copper precipitated was determined as cuprous oxide, as cupric oxide and by Low's method.

In the following table all the results arranged in the same horizontal row were obtained with the same solution and give the averages of several experiments:

\begin{tabular}{|c|c|c|c|c|c|c|c|c|}
\hline \multicolumn{3}{|c|}{$\mathrm{Cu}$ from $\mathrm{Cu}_{2} \mathrm{O}$. } & \multicolumn{3}{|c|}{$\mathrm{Cu}$ from CuO. } & \multicolumn{3}{|c|}{ Cu by r.ow } \\
\hline Clar. & Unclar. & Diff & Clar. & Enclar. & Diff. & Clar. & Unclar. & Diff \\
\hline 0.2724 & 0.2789 & 0.0065 & 0.2706 & $0.275^{8}$ & $0.005^{2}$ & 0.2660 & 0.2720 & 0.0060 \\
\hline 0.2750 & 0.2800 & 0.0050 & 0.2712 & 0.2745 & 0.0033 & 0.2660 & 0.2680 & 0.0020 \\
\hline $0.28+5$ & 0.2857 & 0.0012 & $0.28 I_{2}$ & 0.2819 & 0.0007 & 0.2718 & 0.2735 & 0.0017 \\
\hline 0.4151 & 0.1220 & 0.0069 & 0.1084 & $0 .+139$ & 0.0054 & 0.4054 & 0.4078 & 0.0024 \\
\hline Averige & ilierence & 0.0049 & & & 0.0037 & & & 0.0030 \\
\hline
\end{tabular}

If we compare the figures for the clarified and unclarified solutions, we find that they are invariably higher in the latter case. The difference is largest where the precipitate was weighed as cuprous oxide, and smallest where it was weighed as metallic copper.

If we now compare the difference in the weight of copper by the three methods, we find the following:

clarified.

$\begin{array}{cc}\begin{array}{c}\text { Difference between } \\ \text { Cu from Cu }\end{array} & \begin{array}{c}\text { Diference between } \\ \text { Cu from CuO } \\ \text { and cuby Low. }\end{array} \\ 0.0018 & 0.0046 \\ 0.0038 & 0.0052 \\ 0.0033 & 0.0094 \\ 0.0067 & 0.0030\end{array}$

Average, 0.0039

$\begin{array}{cc}\begin{array}{c}\text { Difference between } \\ \begin{array}{c}\text { Cufrom } \mathrm{Cu}_{2} \mathrm{O} \\ \text { and Cu from CuO. }\end{array}\end{array} & \begin{array}{c}\text { Difference between } \\ \text { Cu from CuO } \\ \text { and Cu by Low. }\end{array} \\ 0.003 \mathrm{I} & 0.0038 \\ 0.0055 & 0.0065 \\ 0.0038 & 0.0084 \\ 0.008 \mathrm{I} & 0.006 \mathrm{I}\end{array}$

$0.005 \mathrm{I}$

0.0062

The difference between the $\mathrm{Cu}$ from $\mathrm{Cu}_{2} \mathrm{O}$ and $\mathrm{Cu}$ from $\mathrm{CuO}$ is partly due to the same cause as in the analysis of pure invert sugar solutions. The remainder must be ascribed to the precipitation of organic matter. More organic matter is precipitated from the unclarified than from the clarified solutions, as has been anticipated. The difference between the $\mathrm{Cu}$ from $\mathrm{CuO}$ and the $\mathrm{Cu}$ by Low's method is due to the precipitation 
of mineral matter. This is in some cases higher in the clarified than in the unclarified solution, in other cases lower.

The results very clearly show the necessity of determining the copper in the precipitate, when analyzing low grade products. If this be done, then only the copper reducing substances are determined. But even then there remains a difference between the clarified and the unclarified solution, the former giving lower results. We know that neutral lead acetate does not remove reducing sugars. The difference must therefore be due to the precipitation of other reducing substances by the neutral lead acetate. Whether any of these remain in solution, thus increasing the apparent percentage of reducing sugars, cannot be decided with our present knowledge of the non-sugars occurring in molasses. However, since the average difference in the reduced copper between the clarified and unclarified solutions was only small, these reducing non-sugars cannot be present in appreciable quantities in the low grade molasses investigated.

\section{Summary.}

The time consumed by a determination of reducing sugars with the method of Munson and Walker may be shortened appreciably by filtering the precipitate through a Neubauer crucible instead of a porcelain Gooch and weighing the precipitate as cupric oxide after heating for ten minutes over a Bunsen burner. The tables of Munson and Walker can then be used after adding 0.0017 gram to the metallic copper calculated from the oxide.

In the determination of reducing sugars in low grade sugar products the quantity of metallic copper present in the precipitate should always be determined. If that be done, clarification with just a sufficient amount of neutral lead acetate may not be necessary in all cases, but it is advisable to use it, because it will remove at least a part of the reducing non-sugars without affecting the sugars.

The use of a Munne-Neubauer crucible has, in all these cases, no advantage over the asbestos tube or Gooch crucible, because the precipitate must be dissolved again.

It appears that in the analysis of low grade products the volumetric method is preferable to the gravimetric, because the errors produced by the precipitation of organic and mineral matter are avoided, and fairly accurate results may be obtained in much less time. Among the different volumetric methods we have recently made some experiments with that proposed by Ivar Bang. ${ }^{1}$ It does not seem to answer the purpose as well as the usual method. The solution employed contains too little copper sulphate per liter, and it is impossible to dissolve more with-

'Biochem. Z., 2, 271. 
out changing the quantities of the other ingredients. If this be done, the precipitate obtained is not pure white. Moreover, the end-point is not sharp enough when highly colored products are analyzed.

\section{DETERMINATION OF SUGAR IN MEATS. ${ }^{1}$}

By A. Lowenstein aND W. P. DUNNE.

Received July 21, 1908.

The determination of "Reducing Sugar" in meats as published in several bulletins of the United States Department of Agriculture and the Association of Official Agricultural Chemists is substantially as follows:

"Boil 100 grams of the finely divided meat for 15 or 20 minutes in a 500 cc. graduated flask, with a convenient volume of water. Add a few cubic centimeters of normal lead acetate, cool to room temperature, make up to the mark with water, and filter through a folded filter. Remove the lead, and determine reducing sugar as $d$-glucose, etc." (Bulletin ro7, Bureau of Chemistry, page I I I).

Upon considering this method one will note that there are Ioo grams of meat present in a $500 \mathrm{cc}$. flask and in addition a voluminous precipitate which results upon the addition of lead acetate. It is obvious at a glance that to use a portion of the filtrate from this mixture and regard it as an aliquot part involves an error, which in the present method is of considerable magnitude, inasmuch as the percentage of reducing sugar present in meats is usually small, so that the error involved becomes of considerable importance. As this is the only method on record known to the writers for the determination of sugar in meats, its use is naturally also extended to the determination of sucrose in meats as well, and here the same error is involved, the magnitude of which is shown in Tables I, II and III. We find the method as outlined above to be rather difficult to manipulate, particularly in the case of meats of high fat content, such as bacon. The introduction of 100 grams of meat into a 500 cc. flask is tedious, when a large number of determinations are to be made; filtration after clarifying with lead acetate is difficult and the use of the lead salt causes a precipitate error, and another precipitate error is involved on removing the excess of lead.

In view of the above we desire to offer a method which has been used satisfactorily in this laboratory, the principal advantages of which are as follows: That it eliminates the error indicated above; that one can conveniently and accurately determine the percentage of nitrates (saltpeter) in the same portion; that it is not necessary to tise clarifying agents,

${ }^{1}$ Read before the Agriculture and Food Sections of the American Chemical Society at the New Haven Meeting. 